\title{
EFFECT OF RIDGE SPLITTING SIMULTANEOUS WITH IMPLANT PLACEMENT FOR TREATMENT OF NARROW RIDGE
}

\author{
Ahmed A Abd Elhalim*, Bahaa Tawfik**, Ahmed Hosni ${ }^{* * *}$
}

\begin{abstract}
Objectives: To assess the effect of ridge splitting simultaneous with implant placement for treatment of narrow ridges. Subjects and Methods: Twenty four implants were placed in twelve patients with narrow alveolar ridge of maxilla randomly selected from the outpatient clinic of the Department of Oral and Maxillofacial Surgery, Faculty of Dental medicine Cairo (Boys) Al-Azhar University. Results: Ridge splitting showed increase in bone width, bone density and bone stability with minimal pain and postoperative swelling. Conclusions: Ridge splitting is a reliable method for treatment of narrow ridge.
\end{abstract}

Keywords: Ridge splitting, narrow ridge, implant.

\section{INTRODUCTION}

Tooth loss may occur gradually throughout life span, replacement of missing teeth with dental implant procedures is one of the greatest advances in dentistry. The problem of resorbed ridges and the ways to add hard and soft tissues in defective sites to provide adequate height and width for appropriate implant insertion has still remained a challenge ${ }^{(1)}$.

Alveolar ridge volume reduction is a direct consequence of tooth loss ${ }^{(2)}$. This dimensional change occurs mainly at the expense of bone remodeling ${ }^{(3)}$. The bone resorption can make it impossible to insert implants due to inadequate space or can create unfavorable aesthetic and functional conditions for prosthetic rehabilitation ${ }^{(4)}$.

Different ridge augmentation techniques have been utilized with comparable success to regain bone thickness in resorbed ridges. These include bone grafting procedures such as guided bone regeneration $(\mathrm{GBR})^{(5)}$, onlay block graft, ${ }^{(5)}$ distraction osteogenesis ${ }^{(6)}$, and inlay graft ${ }^{(7)}$. Guided bone regeneration show relatively long healing time and the risk of membrane exposure that could result in bone loss or implant failure are the major limitations of this technique ${ }^{(8,9)}$. On the other hand, onlay bone graft show relevant morbidity, and the resorption of a significant part of the graft or its exposure are two of the most frequently reported complications $^{(10,11)}$.

As a result of these drawbacks, another treatment option for augmentation of the buccolingual dimension of the alveolar ridge is ridge split technique. This technique is performed by using Osteotomes, chisels, horizontal spreaders or screw spreaders to create a controlled greenstick fracture and split the alveolar ridge into 2 parts in order to create a wider implant bed. After implant placement, the space between the implant periphery and surrounding bone is called the gap or jumping distance, it was once believed that if this distance was greater than $1.5 \mathrm{~mm}$, a bone graft was needed; however, this is controversial and many clinicians believe that larger gap fills with bone ${ }^{(12,13)}$.

\footnotetext{
* B.D.S 2009, Faculty of Dental Medicine, Tanta University, Dentist at Ministry of Health

** Assistant Professor of Oral and Maxillofacial Surgery, Faculty of Dental Medicine,Boys,Cairo Al-Azhar University *** Lecturer, Department of Oral and Maxillofacial Surgery Faculty of Dental Medicine,Boys,Cairo Al-Azhar University
} 
The ridge splitting technique requires a minimum of $3 \mathrm{~mm}$ of bucco-lingual width with at least $1 \mathrm{~mm}$ of cancellous bone between the 2 cortical plates, which would allow introduction of instruments and the maintenance of good blood supply to the split part ${ }^{(14)}$.

\section{SUBJECTS AND METHODS}

This study was carried out on twelve patients selected from the Outpatients Clinic of Oral and Maxillofacial Surgery, Faculty of Dental Medicine, Al-Azhar University.

\section{Clinical inclusion criteria:}

1. Patients diagnosed with narrow ridges $(3-6 \mathrm{~mm})$ in maxilla as determined by cone beam CT.

2. Patient cooperation and motivation

3. Good oral hygiene.

4. Patients age ranged between (18 to 50) from both sexes.

\section{Preoperative evaluation}

Brief history was recorded and a clinical examination was done. Preoperative CBCT was taken for every patient.

\section{Surgical procedure:}

\section{A) Disinfections \& Anaesthetic Technique:}

Disinfection was done with Betadiene for 5 minutes. The patient instructed to rinse his mouth with Chlorhexidine Gluconate $0.12 \%$ (Orovex: Oral Rinse/Macro Company) mouthwash prior to the surgery. Anaesthesia was performed by Mepevacine Hcl 2\% (Vasoconstrictors Levonordefrine 1: 20000 Alex Company) for anesthesing of the site of the surgery.

\section{B) Surgical flap exposure:}

A mucoperiosteal flap extending from the mesial to the distal tooth related to the surgical site was reflected to obtain access and visibility to the subperiosteal surgical site using a sharp \#15 blade.

\section{C) Ridge split \& expansion technique:}

An axial osteotomy of the edentulous alveolar ridge was initiated at the center of the ridge using a surgical disc mounted on low speed handpiece. Chiesel then used to split the alveolar ridge into buccal and palatal tables till desired osteotomy width was obtained. The osteotomy width was measured by using periodontal probe ${ }^{(15)}$.

Bone expanders of increasing diameters were used between the bone tables to make expansion of bone until the desired horizontal ridge dimension was obtained. This whole step was done by using (Split Master II Kit, MR.CURETTE Company, South Korea) for splitting and expansion of the alveolar ridge.

\section{D) Implant installation:}

The implant (Biomate Medical Devices Technology Company, Taiwan) was installed in the prepared site manually till primary friction was obtained. Wrench ratchet was then used to exert more force to seat implant in place to full length.

\section{E) Flap Repositioning \&Closure:}

Flap was repositioned in its place and soft tissue had been closed over the implant using (proline) 3-0 suture.

\section{Postoperative care:}

\section{1) Immediate Postoperative care:}

Patients were instructed to take a postoperative medical regimen containing the following:

1. Augmentin 1 gm (Amoxicillin $875 \mathrm{mg}+$ clavulinic acid 125mg GSK Company) capsules ; 1 cap. every 12 hours for 7 days.

2. Brufen $600 \mathrm{mg}$ (Ibuprofene, Abbot) tablet 1 tab. every 8 hours for 3 days.

3. Alphintern $10 \mathrm{mg}$ (Amoun Pharmaceuticals) tablet; 1 tab. every 8 hours for 7 days, one hour before each meal. 
4. Chlorhexidine gluconate $12 \%$ mouthwash (Chymotrypsin + Trypsin, Amoun Company) 2 times daily for 7 days.

\section{2) Postoperative instructions:}

\section{$1^{\text {st }}$ day instructions:}

Instruct the patient to place cold fomentation, never to dink hot drinks, never to smoke and never to use any mouth wash.

\section{$2^{\text {nd }}$ day instructions:}

Stopping the cold fomentation and mouth rinsing with Orovex mouth wash \& sterile saline.

\section{$3^{\text {rd }}$ day instructions:}

Hot fomentation ( 10 minutes on every 30 minutes, at least 8 times a day)

\section{3) Patient Follow up:}

The first visit was to check the site of surgery, facial swelling and assure that patients followed the given instructions. The second visit was at the $4^{\text {th }}$ day to check facial swelling. The third visit was at the 7th day after surgery to check facial swelling and to remove the stitches.

\section{RESULTS}

\section{1- Pain (visual analogue scale):}

All patients experienced mild pain to moderate pain at the surgical site. Seven patients experienced mild pain while five patients experienced moderate pain. In the seventh day, the same patients were evaluated by pain severity, all patients experienced no pain to mild pain. Five patients experienced mild pain while seven patients experienced no pain at all. There was statistically significant decrease of pain intensity after seven days.

\section{Implant stability:}

Immediately after implant insertion, the mean value of ISQ was 60.82 in the first stage. At 6 months the mean value increased to 72.17 . There was statistically significant increase of implant stability after 6 months.

\section{Bone width:}

The alveolar ridge width range was (3-6) $\mathrm{mm}$ with mean value was $4.06 \mathrm{~mm}$. The mean value of the bone width after 6 months was $7.17 \mathrm{~mm}$. There was statistically significant increase of bone width after 6 months.

\section{Bone Density}

The bone density around implants by Hounsfield Unit (HU) was obtained from the CBCT preoperative with mean value of $546 \mathrm{HU}$ and 6 months postoperatively with mean value $675 \mathrm{HU}$. There was a statistically significant increase of bone density after 6 months.

\section{Facial swelling:}

There was statistically significant increase of facial swelling 1 day postoperatively, followed by statistically significant decrease after 4 days, followed by statistically insignificant decrease after 7 days. There was no statistically significant difference between preoperative and 1 week postoperative facial measurements for swelling.

\section{DISCUSSION}

There are many advantages of ridge splitting procedure. It eliminates the requirement of harvesting bone from a second surgical site as intraoral (ramus , tuberosity, mandibular symphysis ) or extraoral (tibia, iliac crest $)^{(16)}$. Implants placed immediately after the ridge split procedure also reduces patients costs and treatment time ${ }^{(17)}$. The main surgical risk is the fracture of the labial cortical plate which may occur during ridge split or ridge expansion by using bone expanders or during implant insertion ${ }^{(18)}$. In previous studies, the risk of cortical plate fracture is minimal during implant placement ${ }^{(19)}$.

Use of chlorhexidine preoperatively is very important as an antiseptic agent due to its 
antimicrobial activity and its ability to reduce plaque formation at different concentration ${ }^{(20)}$. We also it postoperatively which played indispensable part in achieving satisfactory healing. This was also supported by Tolstunov ${ }^{(21)}$ who clarified the importance of maintaining a meticulous oral hygiene and chlorhexidine rinses in the postoperative healing period.

There was a controversy about the use of bone graft is within the space generated after ridge splitting. Some authors reported that no need for bone graft if this space smaller than $3 \mathrm{~mm}^{(22)}$. However in the present study, there is no need for bone graft as we use bone expanders to the desired width.

In the current study, there was a significant increase in the pain in the first day of implant placement which became minimal at seventh day similar to the study performed by Ramal et al ${ }^{(23)}$ and Moro et $\mathrm{al}^{(24)}$ that showed that soft tissue healing after ridge splitting was uneventful as well as the pain and swelling were comparable to usual dentoalveolar surgical procedures.

Usually the primary stability of the dental implant originates from mechanical engagement with the cortical bone. It is affected by the quantity, quality of bone that the dental implant was inserted into, surgical procedure, length, diameter, and implant form. On the other hand secondary stability is considered as a biological phenomenon, on which it's a result of healing that takes place around the implant itself ${ }^{(25)}$.

The results of the present study revealed that the implant stability increased significantly after 6 months which was similar to study by Kreissel ${ }^{(26)}$ who assessed the implant stability in expanded ridges and reported that the application of the bone spreaders significantly increased ISQ values over the study period.

Digholkar et al ${ }^{(27)}$ specified the importance of ISQ where the acceptable stability range, based on many studies made with RFA, lies between 55 and 85 ISQ with an average ISQ level of 70. In the present study, the mean ISQ value after a healing period of 6 months was 72.12 .

In the present study, there was significant increase in bone width due to alveolar bone splitting and expansion resulted in an indication of a successful treatment which was similar to the study performed by Simion et al ${ }^{(28)}$ and Scipioni et al ${ }^{(29)}$. They demonstrated an alveolar width gain between 1 and $4 \mathrm{~mm}$ after the split-crest procedure and successful immediate implant placement.

In the current study, bone density around implant measured by Hounsfield Unit (HU) showed highly statically significant difference between preoperative $(546 \mathrm{HU})$ and postoperative $(650 \mathrm{HU})$ CBCT.

These results correlated with the studies conducted by Fanuscu et al ${ }^{(30)}$ where expansion technique resulted in increase in bone density compared with drilling technique which aid in better primary stability of the implant as the implant adheres to bone firmly without mobility.

In the current study, there was significant increase in the facial swelling at the first day which decreases gradually similar to the study by Rahal ${ }^{(31)}$ who placed 20 implants in narrow ridge of anterior maxilla by using bone expanders. Also similar to the study made by Ramal ${ }^{(23)}$ who placed 20 implants in posterior mandible by using ridge split technique.

The least postoperative swelling and complications were due to using of series of chisels and mallet which lead to less resonance frequency, less heat generation, less bone loss and higher implant stability ${ }^{(23)}$.

\section{CONCLUSIONS}

1. Ridge splitting technique with simultaneous immediate implant placement is a successful minimally invasive technique for horizontal augmentation of the maxillary narrow alveolar ridge. 
2. Ridge splitting and bone expanders lead to increase in the stability of the dental implant and density around dental implant.

3. Ridge splitting technique show minimal postoperative pain and facial swelling.

\section{REFERENCES}

1. Chiapasco M, Zaniboni M, Boisco M. Augmentation procedures for the rehabilitation of deficient edentulous ridges with oral implants. Clin Oral Implants Res. 2006; 17:136-59.

2. Demarosi F, Leghissa G, Sardella A, Lodi G, Carrassi A. Localised maxillary ridge expansion with simultaneous implant placement: a case series. Br J Oral Maxillofac Surg Res. 2009; 47:535-40.

3. Barone A, Covani A. Maxillary alveolar ridge reconstruction with nonvascularized autogenous block bone: clinical results. J Oral Maxillofac Surg Res.2007; 65:2039-46.

4. Bidra A. Surgical and prosthodontic consequences of inadequate treatment planning for fixed implant-supported prosthesis in the edentulous mandible. Journal of Oral and Maxillofacial Surgery Res 2010; 68:2528-36.

5. Chiapasco M, Abati S, Romeo E, Vogel G. Clinical outcome of autogenous bone blocks or guided bone regeneration with e-PTFE membranes for the reconstruction of narrow edentulous ridges. Clin Oral Implants Res. 1999; 10:278-88.

6. Takahashi T, Funaki K, Shintani H, Haruoka T. Use of horizontal alveolar distraction osteogenesis for implant placement in a narrow alveolar ridge: A case report. Int J Oral Maxillofac Implants Res 2004; 19:291-4.

7. Kahnberg K, Nystrom E, Bartholdsson L. Combined use of bone grafts and Brånemark fixtures in the treatment of severely resorbed maxillae. Int J Oral Maxillofac Implants. 1989; 4:297-304.

8. Machtei E. The effect of membrane exposure on the outcome of regenerative procedures in humans: a meta-analysis. J Periodontol, 2001; 72:512-16.

9. Nyström E, Ahlqvist J, Kahnberg K, Rosenquist J. Autogenous onlay bone grafts fixed with screw implants for the treatment of severely resorbed maxillae. Int J Oral Maxillofac Surg, 1996; 25:351-9.

10. Maiorana C, Beretta M, Salina S, Santoro F. Reduction of autogenous bone graft resorption by means of bio-oss coverage: a prospective study. Int J Periodontics Restorative Dent. 2005; 25:19-25.
11. Proussaefs P, Lozada J. The use of intraorally harvested autogenous block grafts for vertical alveolar ridge augmentation: a human study. Int J Periodontics Restorative Dent. 2005; 25:351-63.

12. Botticelli D, Berglundh T, Buser D, Lindhe J. The jumping distance revisited: An experimental study in the dog. Clin Oral Implants Res. 2003; 14:35-42.

13. Wang H, Boyapati L. Principles for predictable bone regeneration. Implant Dent. 2006; 15:8-17.

14. Dene L, Condos S. Ridge expansion and immediate implant placement in the esthetic zone. N Y State Dent J. 2010; 76:28-31.

15. Mechery, R., Thiruvalluvan, N., \& Sreehari, A. K. (2015). Ridge split and implant placement in deficient alveolar ridge: Case report and an update. Contemporary clinical dentistry, 6(1), 94-97.

16. Basa S, Varol A, Turker N. Alternative bone expansion technique for immediate placement of implants in the edentulous posterior mandibular ridge: A clinical report. Int J Oral Maxillofac Implants. 2004; 19:554-8.

17. Lindquist L, Rockler B, Carlsson G. Bone resorption around fixtures in edentulous patients treated with mandibular fixed tissue-integrated prostheses. The Journal of prosthetic dentistry. 1988 Jan 1; 59(1):59-63.

18. Torella F,Pitarch J, Cabanes G, Anitua E.Ultrasonic osteotomy for the surgical approach of the maxillary sinus: a technical note.International Journal of Oral \& Maxillofacial Implants.1998; 13: 697-700.

19. Blus C,Szmukler S.The split crest procedure with ultrasonic bone surgery.Results of a 3 years experience with 172 treated sites.Clinical Oral Implants research. 2004; 14:36-37.

20. Sjostrom M, Sennerby L, Nilson H, Lundgren S. Reconstruction of the atrophic edentulous maxilla with free iliac crest grafts and implants: A 3-year report of a prospective clinical study. Clin Implant Dent Rela Res. 2007;9(1):46-59.

21. Tolstunov L. Ridge-split procedure for horizontal augmentation of the alveolar ridge in implant dentistry. Oral Maxillofac Surg 2013;21:1-55

22. Kim Y, Kim S. Horizontal ridge expansion and implant placement using screws: a report of two cases. J Korean Assoc Oral Maxillofac Surg 2014; 40:233-9.

23. Ali Rammal. Modified Staged Ridge Splitting Technique Versus Conventional Technique for Horizontal Expansion of Narrow Posterior Mandible (Randomized Controlled Clinical Trial. Acta Scientific Dental Sciences 2.7 (2018): 101-9. 
24. Moro A. Alveolar Ridge Split Technique Using Piezosurgery with Specially Designed Tips. BioMed Research International 2017; 45:303-78.

25. Park J, Lee J, Kim S, Lee J. A comparison of implant stability quotients measured using magnetic resonance frequency analysis from two directions: a prospective clinical study during the initial healing period. Clinical Oral Implant Research. 2010; 21(6):591-7.

26. Kreissel P, Kölpin F, Graef F, Wichmann M, Karl M. Effect of rotating osteotomes on primary implant Stability-an in vitro investigation. J Oral Implantol. 2013; 39(1):52-7.

27. Digholkar S, Venu Madhav V, Palaskar J. Methods to measure stability of dental implants. J Dent Allied Sci 2014;3:17-23
28. Simion M, Baldoni M, Zaffe D. Jawbone enlargement using immediate implant placement associated with a splitcrest technique and guided tissue regeneration.The International Journal of Periodontics \& Restorative Dentistry, 1992;12(6) :462-73.

29. Scipioni A, Bruschi G, Calesini G. The edentulous ridge expansion technique: A five-year study. Int J Periodontics Restorative Dent. 1994; 14:451-9.

30. Fanascu M, Chang T, Akca K. Effect of surgical techniques on primary implant stability and peri-implant bone. J Oral Maxillofac Surg. 2007; 65:2487-91.

31. Rahal R. Efficiency of ridge expansion using screw type expanders with simultaneous trabecular implant placement in narrow anterior maxilla.E.D.J 2018; 64(2): 113- 22. 